\title{
TOEPLITZ MATRICES GENERATED \\ BY THE LAURENT SERIES EXPANSION OF AN ARBITRARY RATIONAL FUNCTION
}

BY

\section{K. MICHAEL DAY}

\begin{abstract}
Let $T_{n}(f)=\left(a_{i-j}\right)_{i, j=0}^{n}$ be the finite Toeplitz matrices generated by the Laurent expansion of an arbitrary rational function. An identity is developed for $\operatorname{det}\left(T_{n}(f)-\lambda\right)$ which may be used to prove that the limit set of the eigenvalues of the $T_{n}(f)$ is a point or consists of a finite number of analytic arcs.
\end{abstract}

1. Introduction. Let $f(z)=\Sigma a_{m} z^{m}$ be the Laurent expansion of an arbitrary rational function. Define matrices $T_{n}(f)$ where $T_{n}(f)=\left(a_{i-j}\right), i, j=0, \cdots, n$. Such matrices are called Toeplitz matrices and may be generated by functions which are not rational. Denote by $\sigma_{n}$ the set of $n+1$ eigenvalues of $T_{n}(f)$,

$$
\sigma_{n}=\left\{\lambda_{n 0}, \lambda_{n 1}, \cdots, \lambda_{n n}\right\} \text {. }
$$

Let

$$
B=\left\{\lambda: \lambda=\lim \lambda_{m}, \lambda_{m} \in \sigma_{i_{m}}\right\}
$$

where $i_{1}, i_{2}, \cdots$ is an increasing sequence of integers. A characterization of this set for complex valued functions was initiated in 1960 and was published for the case: $f$ is a Laurent polynomial, $f(z)=\Sigma_{-k}^{h} a_{m} z^{m}, h, k \geqslant 1$ [4]. Let

$$
D^{n}(f-\lambda)=\operatorname{det}\left(T_{n}(f-\lambda)\right) \text {. }
$$

Schmidt and Spitzer employed an identity of Harold Widom which up to a constant factor evaluates the $D^{n}(f-\lambda)$ when $f$ is a Laurent polynomial. We develop an identity for $D^{n}(f-\lambda)$ for $f$ an arbitrary rational function which, using the techniques of Schmidt and Spitzer, allows one to show that $B$ is a point or consists of a finite number of nondegenerate analytic arcs.

For simplification in the proof of the identity and notational convenience we make certain assumptions about the function $f$ which are essentially nonrestrictive and work directly with the determinants $D^{n}(f)$. Due to the complexities of notation, we observe the following convention. "(*)" designates a mathematical expression where " $*$ " is the number of the expression, and "(*)" is used to

Presented to the Society, November 16, 1973; received by the editors December 14, 1973. AMS (MOS) subject classifications (1970). Primary 30A08, 30A16; Secondary 65F15. 
represent the object appearing in that expression.

In order that the Laurent series not reduce to a power series in which case the determinants $D^{n}(f)$ are triangular and the set $B$ reduces to a point we make the following assumptions. Let there be an annulus $A$ with center the origin,

$$
A=\left\{z: R_{1}<|z|<R_{2}, 0 \leqslant R_{1}<R_{2}<\infty\right\} .
$$

Let $D_{k}(z)$ and $F_{h}(z)$ be polynomials of exact degree $k$ and $h$ respectively where the roots of $D_{k}(z)$ lie in the set $|z| \leqslant R_{1}$, and those of $F_{h}(z)$ lie in the set $|z| \geqslant$ $R_{2}$. Let $G_{k+m}(z)$ be a polynomial of exact degree $k+m$ having no common factors with the polynomials $D_{k}(z)$ and $F_{h}(z)$.

Proposition 1.1. Let $k \geqslant 1, m \geqslant \max (1, h)$, and $G_{k+m}(z), D_{k}(z)$, and $F_{h}(z)$ satisfy the conditions given above. If

$$
f(z)=G_{k+m}(z) / D_{k}(z) F_{h}(z)
$$

and

$$
f(z)=\sum_{-\infty}^{\infty} a_{v} z^{v}
$$

is the Laurent series representation of $f(z)$ in the annulus $A$, then there exist positive and negative powers of $z$ which in the above expansion have nonzero coefficients.

Proof. Assume that $k, h \geqslant 1$. If $a_{v}=0$ for all $v \leqslant-1$, then (1.3) is a power series expansion which converges in the set $|z|<R_{2}$. This contradicts the existence of at least one pole in the set $|z| \leqslant R_{1}<R_{2}$. If $a_{v}=0$ for all $v \geqslant 1$, then (1.3) converges in the set $|z|>R_{1}$ contradicting the existence of a pole in the set $|z| \geqslant R_{2}>R_{1}$. In the exceptional case where $h=0$, then $m \geqslant 1$. Consequently $f(z)$ defined by (1.2) has a pole of order $m$ at $z=\infty$. So $a_{m} \neq 0$.

2. Reduction of $D^{n}(f)$ to a determinant of fixed order $m$. Let $f(z)$ satisfy the hypothesis of Proposition 1.1. In addition we assume that the roots of $G_{k+m}(z)$ denoted by $r_{i}, i=1, \cdots, k+m$, are distinct and not equal to zero. We may assume that the coefficient $g_{0}$ of the $z^{k+m}$ term of $G_{k+m}(z)$ is equal to 1 . For if $f(z)$ is divided by $g_{0}$ we have simply divided each $a_{n}$ in the expansion of $f$ by $g_{0}$. So $D^{n}(f)=g_{0}^{n+1} D^{n}\left(g_{0}^{-1} f\right)$. The proof is long and will be broken up into a series of lemmas. We recommend that the reader turn to Theorem 3.1 for the final result before proceeding with the proof. We introduce the following notation.

$$
z^{-(k+m)} G_{k+m}(z)=\prod_{i=1}^{k+m}\left(1-r_{i} z^{-1}\right)=\sum_{i=0}^{k+m} g_{-i} z^{-i}
$$




$$
\begin{gathered}
1 / z^{-(k+m)} G_{k+m}(z)=\sum_{i=0}^{\infty} g_{-i}^{*} z^{-i}, \\
z^{-k} D_{k}(z)=\prod_{i=1}^{k}\left(1-\delta_{i} z^{-1}\right)=\sum_{j=0}^{k} d_{-j}^{*} z^{-j}, \\
1 / z^{-k} D_{k}(z)=\sum_{j=0}^{\infty} d_{-j} z^{-j}, \\
F_{h}(z)=\prod_{i=1}^{h}\left(1-\rho_{i}^{-1} z\right)=\sum_{i=0}^{h} f_{i}^{*} z^{i}, \\
1 / F_{h}(z)=\sum_{i=0}^{\infty} f_{i} z^{i}, \\
z^{-(k+m)} G_{k+m}(z) / z^{-k} D_{k}(z)=\sum_{i=0}^{\infty} e_{-i} z^{-i}, \\
z^{-k} D_{k}(z) / z^{-(k+m)} G_{k+m}(z)=\sum_{i=0}^{\infty} e_{-i}^{*} z^{-i}, \\
f(z)=G_{k+m}(z) / D_{k}(z) \cdot F_{h}(z)=\sum_{-\infty}^{\infty} a_{n} z^{n} .
\end{gathered}
$$

The first two lemmas are devoted to determining certain information about the coefficients of some of these expansions.

LEMMA 2.1. If $g_{-i}^{*}$ is defined by (2.2) and $e_{-i}^{*}$ by (2.9) then $g_{-i}^{*}=$ $\Sigma_{s=1}^{k+m} C_{s} r_{s}^{i}$ holds for $i \geqslant-(k+m)+1$, and $e_{-i}^{*}=\Sigma_{s=1}^{k+m} C_{s} D_{k}\left(r_{s}\right) r_{s}^{i-k}$ holds for $i \geqslant k$ where $C_{s}=r_{s}^{k+m-1} \Pi_{t \neq s}\left(r_{s}-r_{t}\right)^{-1}$.

Proof. By expanding $i / z^{-(k+m)} G_{k+m}(z)$ by partial fractions we obtain the above identity for $g_{-i}^{*}, i \geqslant 0$. In addition, the identity is valid for $i=-1$, $\cdots,-(k+m)+1$ because, for all $i \geqslant-(k+m)+1$,

$$
\sum_{s=1}^{k+m} C_{s} r_{s}^{l}=\sum_{s>t}\left(r_{s}-r_{t}\right)^{-1}\left\|\begin{array}{lllll}
1 & r_{1} & \cdots & r_{1}^{k+m-2} & r_{1}^{i+k+m-1} \\
1 & r_{2} & \cdots & r_{2}^{k+m-2} & r_{2}^{i+k+m-1} \\
\vdots & \vdots & & \vdots & \vdots \\
1 & r_{k+m} & \cdots & r_{k+m}^{k+m-2} & r_{k+m}^{i+k+m-1}
\end{array}\right\|
$$

The identity for the $e_{-i}^{*}$ 's follows from the identity for the $g_{-i}^{*}$ 's and (2.3).

$$
\text { LEMMA 2.2. If }-i+k<0 \text { then } \sum_{j=0}^{k} d_{-j}^{*} b_{-i+j}=0 \text {. }
$$


Proof. The lemma is clearly true if $D_{k}(z)=C z^{k}$ for then, by (2.6) and (2.7), $b_{-i}=0$ for $i>0$. Otherwise expansion of $1 / z^{-k} D_{k}(z) F_{h}(z)$ by partial fractions shows that

$$
b_{-i}=\sum_{j=1}^{k^{\prime}} B_{j} \hat{\delta}_{j}^{i}
$$

where $\hat{\delta}_{j}, j=1, \cdots, k^{\prime}$, are the distinct nonzero roots of $D_{k}(z)$, and $B_{j}$ nonzero constants. Without loss of generality we may assume that all the roots of $D_{k}(z)$ are nonzero. Proof is by induction.

Let $S_{i}(k), i=1, \cdots, k$, be the elementary symmetric functions of the roots $\delta_{i}$ of $D_{k}(z)$ so that from $(2.3)$

$$
\begin{aligned}
\prod_{i=1}^{k}\left(z-\delta_{i}\right) & =d_{0} z^{k}+d_{1} z^{k-1}+\cdots+d_{k} \\
& =z^{k}+S_{1}(k) z^{k-1}+\cdots+S_{k}(k) .
\end{aligned}
$$

Thus to prove the lemma we need to prove that

$$
b_{-i}+S_{1}(k) b_{-i+1}+S_{2}(k) b_{-i+2}+\cdots+S_{k}(k) b_{-i+k}=0 .
$$

Since

$$
\prod_{i=1}^{k-1}\left(z-\delta_{i}\right)\left(z-\delta_{k}\right)=\left[z^{k-1}+S_{1}(k-1) z^{k-2}+\cdots+S_{k-1}(k-1)\right]\left(z-\delta_{k}\right),
$$

it follows that

$$
\begin{aligned}
S_{1}(k) & =S_{1}(k-1)-\delta_{k}, \\
S_{i}(k) & =S_{i}(k-1)-\delta_{k} S_{i-1}(k-1), \quad i=2, \cdots, k-1, \\
S_{k}(k) & =-\delta_{k} S_{k-1}(k-1) .
\end{aligned}
$$

By (2.12) it is clear that the lemma will be proved if we can show that

$$
\begin{array}{r}
\delta_{j}^{t}+\delta_{j}^{t-1} S_{1}(k)+\delta_{j}^{t-2} S_{2}(k)+\cdots+\delta_{j}^{t-k} S_{k}(k)=0, \\
j=1, \cdots, k, t>k .
\end{array}
$$

This is obvious for $k=1$. We need to prove (2.14) for $k+1$. From (2.13) it follows that

$$
\begin{aligned}
\delta_{j}^{t}+ & \delta_{j}^{t-1} S_{1}(k+1)+\cdots+\delta_{j}^{t-k-1} S_{k+1}(k+1) \\
= & \delta_{j}^{t}+\delta_{j}^{t-1} S_{1}(k)+\delta_{j}^{t-2} S_{2}(k)+\cdots+\delta_{j}^{t-k} S_{k}(k) \\
& -\delta_{k+1}\left(\delta_{j}^{t-1}+\delta_{j}^{t-2} S_{1}(k)+\delta_{j}^{t-3} S_{2}(k)+\cdots+\delta_{j}^{t-k-1} S_{k}(k)\right) .
\end{aligned}
$$

By induction, (2.15) equals zero for $j=1, \cdots, k$, and $(\underline{2.15})$ clearly equals zero for $j=k+1$.

Lemma 2.3. $D^{n}(f)$ is equal to $(-1)^{m(n+1-m)}$ multiplied by the determinant of the product of the following three matrices, 


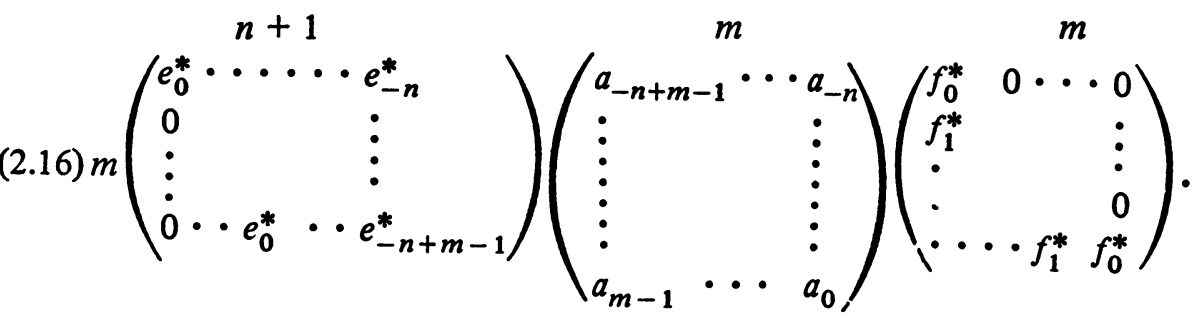

PrOof. In order to prove the above we multiply $D^{n}(f)$ on the left and right by determinants each of which is equal to one. In particular, noting by (2.5) and (2.9) that $e_{0}^{*}=f_{0}^{*}=1$, we multiply $D^{n}(f)$ on the left by the upper triangular determinant,

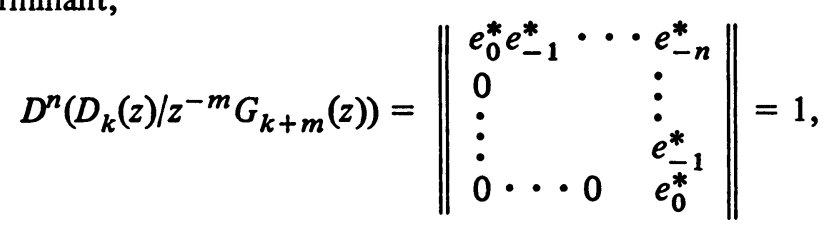

and on the right by the lower triangular determinant,

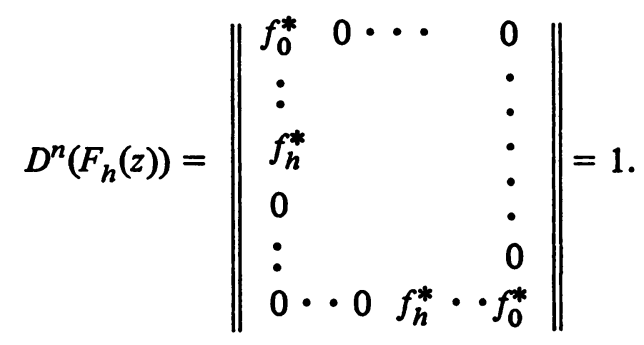

We make the important remark that the above determinants are asymmetric, and that another reduction of $D^{n}(f)$ to a determinant of fixed order is possible. We could have multiplied $D^{n}(f)$ on the left by the upper triangular determinant $D^{n}\left(z^{-k} D_{k}(z)\right)$, and on the right by the lower triangular determinant $D^{n}\left(F_{h}(z) / G_{k+m}(z)\right)$.

Given the relations $(2.1)-(2.10)$ the following may be verified.

$$
\begin{aligned}
& D^{n}(f) \cdot D^{n}\left(F_{h}\right)=\left\|\left(a_{i-j}\right)\right\| \cdot\left\|\left(f_{i-j}^{*}\right)\right\| \\
& =\left\|\begin{array}{llll|ll}
e_{-m} & e_{-m-1} & \cdots & e_{-n} & \cdots & * \\
e_{0} & & \vdots & \vdots & \vdots \\
0 & & \vdots & \vdots & \vdots \\
\vdots & & \vdots & \vdots & \vdots \\
0 & \ldots \ldots & 0 & e_{0} & * & *
\end{array}\right\|
\end{aligned}
$$

where the " $*$ " indicates entries which are left undetermined. Consequently 


$$
\begin{aligned}
& D^{n}(f)=D^{n}\left(D_{k} / z^{-m} G_{k+m}\right) \cdot\left[D^{n}(f) \cdot D^{n}\left(F_{h}\right)\right] \\
& =\left\|\left(e_{i-j}^{*}\right)\right\| \cdot\left[\left\|\left(a_{i-j}\right)\right\| \cdot\left\|\left(f_{i-j}^{*}\right)\right\|\right]
\end{aligned}
$$

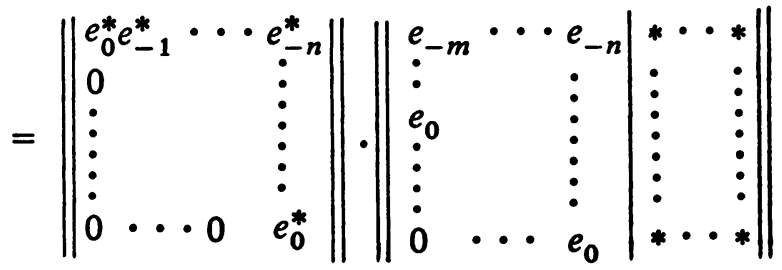

$$
\begin{aligned}
& =\left\|\begin{array}{c|c||}
0 & K \\
\hline I & N
\end{array}\right\|_{n+1-m}^{m} \\
& n+1-m \quad m
\end{aligned}
$$

where $I$ is the identity matrix and $K$ equals the product (2.16). By shifting the rows of $I$ in (2.19) into the upper left-hand corner it follows that

$$
D^{n}(f)=(-1)^{m(n+1-m)}\|K\|,
$$

and the lemma follows.

In the following two lemmas we show that each of the two matrices on the left-hand side of (2.16) may be written as the product of a pair of matrices.

LEMMA 2.4 .

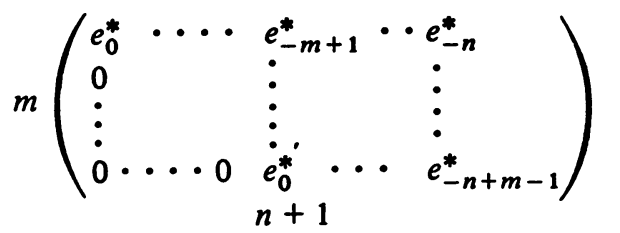

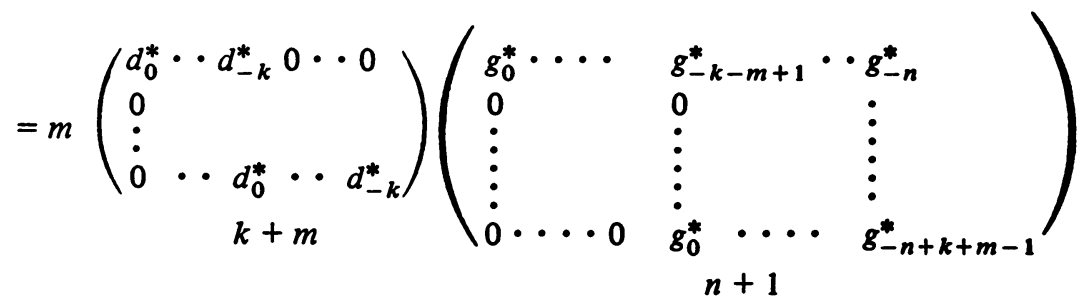

$=A \cdot B$, say.

Proof. Verify by referring to (2.2), (2.3), and (2.9). 
LEMMA 2.5 .

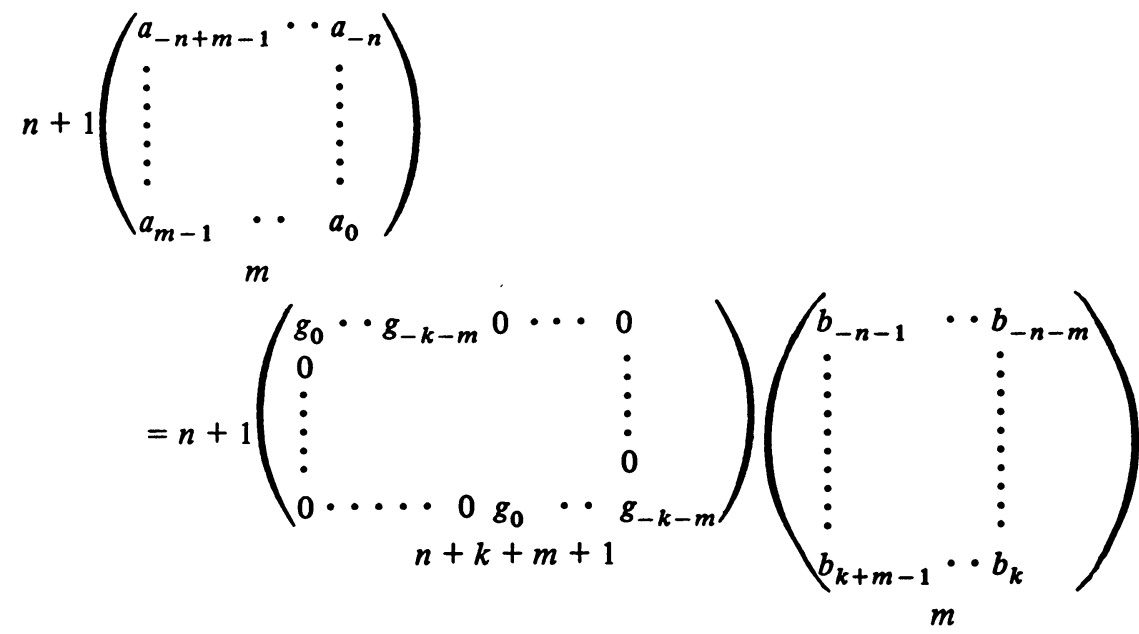

$=C \cdot D$, say.

Proof. Verify by referring to (2.1), (2.7) and (2.10).

It follows from Lemmas 2.4 and 2.5 that if we denote the right-hand matrix of (2.16) by $E$, then the matrix $K$ which equals (2.16) may be written $K=$ $A B C D E$, where

$$
\begin{array}{ll}
A=\left(d_{i-j}^{*}\right), & B=\left(g_{i-j}^{*}\right), \quad C=\left(g_{i-j}\right), \\
D=\left(b_{i-j}\right), & E=\left(f_{i-j}^{*}\right),
\end{array}
$$

as appears in these two lemmas. So by (2.20) it follows that

$$
D^{n}(f)=(-1)^{m(n+1-m)}\|A \cdot B \cdot C \cdot D \cdot E\|,
$$

and we must simplify the product of these matrices whose orders are increasing with $n$.

LEMMA 2.6.

$B \cdot C \cdot D=k+m\left(\begin{array}{lll}b_{-n-1} & \cdots & b_{-n-m} \\ \vdots & & \vdots \\ \vdots & & \vdots \\ b_{-n+k+m-2} & \cdots & b_{-n+k-1}\end{array}\right)$

$m$

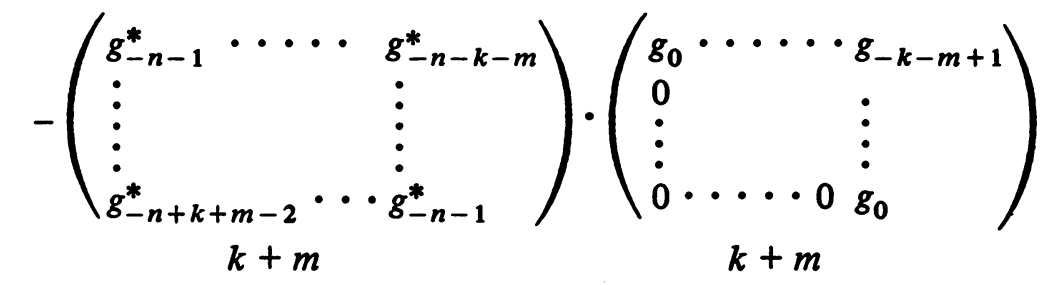


(2.22) (cont.)

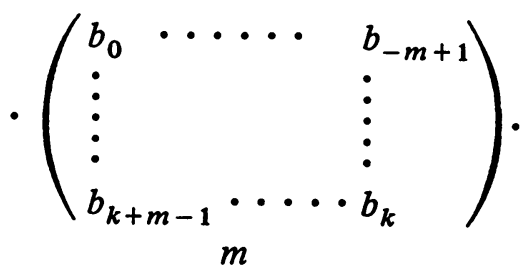

Proof. Multiply $B$ and $C$ together and get that

$$
\begin{aligned}
& B \cdot C=\left(\begin{array}{lllll}
0 & 0 & \cdots & 0 & 0 \\
\vdots & \vdots & & : & : \\
0 & 0 & \cdots & 0 & 0
\end{array} \mid \quad M\right) k+m \\
& k+m \quad n-k-m+1 \quad k+m
\end{aligned}
$$

where $I$ is the identity matrix, and

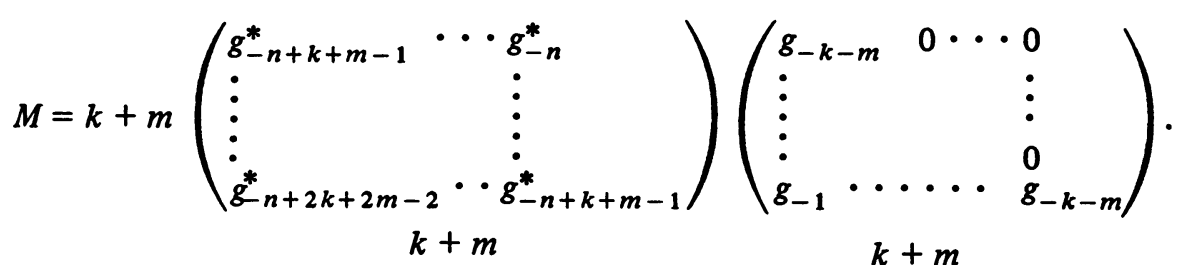

Using the relations (2.1) and (2.2) that $\sum_{i=0}^{\min (v, k+m)} g_{-i} g_{-v+i}^{*}=0$ for $v \geqslant 1$, we may rewrite the matrix $M$ so that

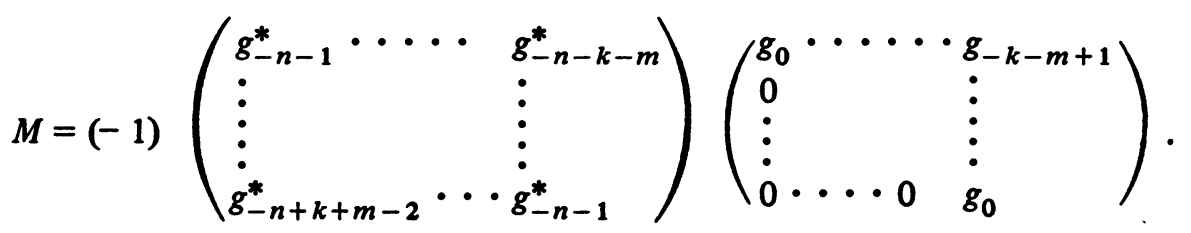

Let $B \cdot C=H$. Multiply $H$ by $D$. We get that

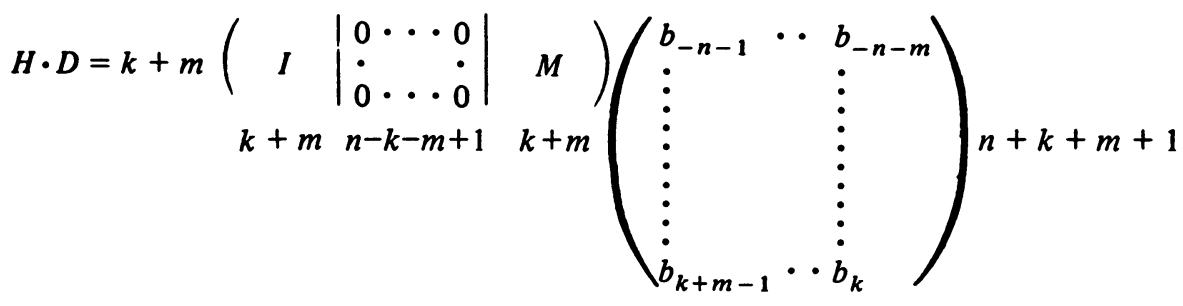

$=(\underline{2.22})$, the desired result. 
LEMMA 2.7.

$$
\begin{aligned}
& A \cdot B \cdot C \cdot D=(-1)\left(\begin{array}{lll}
e_{-n-1}^{*} & \cdots & e_{-n-k-m}^{*} \\
\vdots & & \vdots \\
\vdots & & \vdots \\
e_{-n+m-2}^{*} & \cdots & e_{-n-k-1}^{*}
\end{array}\right) \cdot\left(\begin{array}{ll}
g_{0} \cdots g_{-k-m+1} \\
\vdots \\
\vdots & \\
0 & \cdots g_{0}
\end{array}\right) \\
& k+m \\
& k+m \\
& \left(\begin{array}{lll}
b_{0} & \cdots & b_{-m+1} \\
\vdots & \vdots \\
b_{k+m-1} & \cdots & b_{k}
\end{array}\right) k+m
\end{aligned}
$$

$m$

Proof. We multiply $H \cdot D$ on the left by $A$ where $A$ is the $\left(d_{i-j}^{*}\right)$ matrix of Lemma 2.4. The left-hand component of $H \cdot D$ is the $\left(b_{i-j}\right)$ matrix of (2.20). By Lemma 2.2

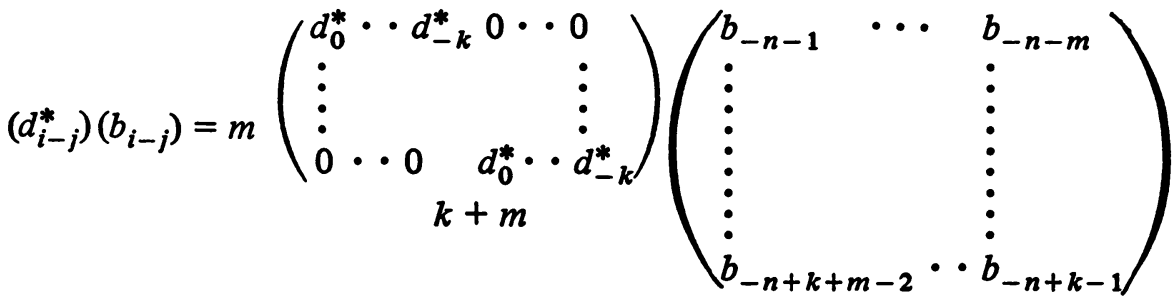

$$
\begin{aligned}
& =m\left(\begin{array}{ccc}
0 & \cdots & 0 \\
\vdots & & \vdots \\
0 & \cdots & 0
\end{array}\right)
\end{aligned}
$$

$m$

We remark that without this result the identity we obtain for $D^{n}(f)$ would not be possible. Continuing our multiplication of $H \cdot D$ by $A$, since by (2.2), (2.3) and (2.9)

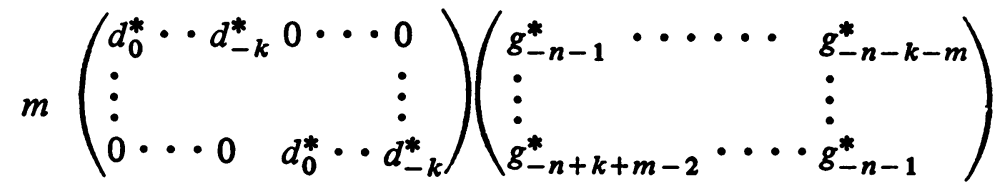

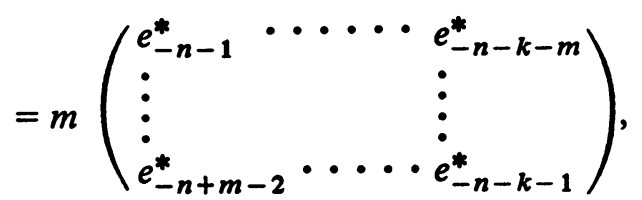

$$
\begin{aligned}
& k+m
\end{aligned}
$$


it follows that $A \cdot B \cdot C \cdot D=A \cdot H \cdot D=(2.23)$.

THEOREM 2.1 .

(2.24)

$$
D^{n}(f)=(-1)^{m(n+1)} \operatorname{det}\left(\begin{array}{lll}
e_{-n-1}^{*} & \cdots & e_{-n-k-m}^{*} \\
\vdots & & \vdots \\
e_{-n+m-2}^{*} & \cdots & e_{-n-k-1}^{*}
\end{array}\right)
$$

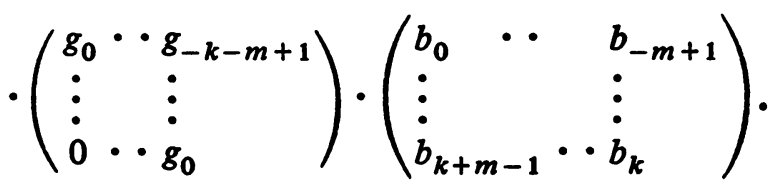

Proof. Since $\|E\|=1,\|A \cdot B \cdot C \cdot D \cdot E\|=\|A \cdot B \cdot C \cdot D\|$. Since $(-1)^{m(n+1-m)}=(-1)^{m(n+1)-m}$, by (2.21) and (2.23) it follows that $D^{n}(f)=$ (2.24). So $D^{n}(f)$ equals a determinant of fixed order $m$.

3. Evaluation of the determinants $D^{n}(f)$. In the last section we showed that $D^{n}(f)$ equaled the determinant of the product of three matrices whose orders were independent of $n$ (2.24). In this section we will evaluate this product. We have of course by (2.1) that $G_{k+m}(z)=\Pi_{i=1}^{k+m}\left(z-r_{i}\right)$. In general, let

$$
G_{k+m}^{S}(z)=\prod_{i=1 ; i \notin S}^{k+m}\left(z-r_{i}\right)
$$

where $S$ is a subset of the integers $(1,2, \cdots, k+m)$. In the event we wish to be explicit about the entries in $S$ we will write $\left(i_{1}, i_{2}, \cdots, i_{\alpha}\right)$ to indicate that $S$ contains the integers $i_{1}, i_{2}, \cdots, i_{\alpha}$. In particular if $S$ consists of the singleton $i$, we write

$$
G_{k+m}^{(i)}(z)=\left(z-r_{i}\right)^{-1} G_{k+m}(z)=\prod_{j=1 ; j \neq i}^{k+m}\left(z-r_{j}\right)
$$

In an analogous way we define the coefficient $a_{m}^{S}$ by means of

$$
\begin{aligned}
& \sum_{-\infty}^{\infty} a_{m}^{S} z^{m}=G_{k+m}^{S}(z) / D_{k}(z) F_{h}(z) \quad \text { and } \\
& \sum_{-\infty}^{\infty} a_{m}^{(i)} z^{m}=G_{k+m}^{(i)}(z) / D_{k}(z) F_{h}(z) .
\end{aligned}
$$

LEMmA 3.1. The product of the three matrices of (2.24), $\left(e_{-n-1+i-j}^{*}\right)$ $\cdot\left(g_{i-j}\right)\left(b_{i-j}\right)$, is equal to the $m$ by $m$ matrix

$$
\left[\sum_{i=1}^{k+m} C_{i} D_{k}\left(r_{i}\right) r_{i}^{n+2-k-s} a_{m-t}^{(i)}\right]
$$

for $s, t=1,2, \cdots, m$, and where $C_{i}=r_{i}^{k+m-1} \Pi_{j \neq i}\left(r_{i}-r_{j}\right)^{-1}$. 
Proof. By Lemma 2.1, $e_{-n}^{*}=\sum_{i=1}^{k+m} C_{i} D_{k}\left(r_{i}\right) r_{i}^{n-k}$. By definition

$$
z^{-(k+m)} G_{k+m}(z)=\prod_{i=1}^{k+m}\left(1-r_{i} z^{-1}\right)=\sum_{i=0}^{k+m} g_{-i} z^{-i}
$$

Consequently

$$
g_{0}=1, g_{-1}=-\sum_{s=1}^{k+m} r_{s}, g_{-2}=\sum_{1 \leqslant s<t}^{k+m} r_{s} r_{t} \text {, etc. }
$$

If we multiply the top row of the $\left(e_{-n-1+i-j}^{*}\right)$ matrix of (2.24) by the columns of the $\left(g_{i-j}\right)$ matrix of (2.24), the following may be easily verified.

$$
\begin{aligned}
e_{-n-1}^{*} g_{0} & =\sum_{i=1}^{k+m} C_{i} D_{k}\left(r_{i}\right) r_{i}^{n+1-k}, \\
e_{-n-1}^{*} g_{-1}+e_{-n-2}^{*} g_{0} & =-\sum_{i=1}^{k+m} C_{i} D_{k}\left(r_{i}\right) r_{i}^{n+1-k} \cdot \sum_{s=1 ; s \neq i}^{k+m} r_{s}, \\
e_{-n-1}^{*} g_{-2}+e_{-n-2}^{*} g_{-1}+e_{-n-3}^{*} g_{0} & =\sum_{i=1}^{k+m} C_{i} D_{k}\left(r_{i}\right) r_{i}^{n+1-k} \cdot \sum_{1<s<t ; s, t \neq i}^{k+m} r_{s} r_{t},
\end{aligned}
$$

and similarly for the remaining products. Thus the top row of $\left(e_{-n-1+i-j}^{*}\right)$ - $\left(g_{i-j}\right)$ is exactly

$$
\left(\sum_{i=1}^{k+m} C_{i} D_{k}\left(r_{i}\right) r_{i}^{n+1-k},-\sum_{i=1}^{k+m} C_{i} D_{k}\left(r_{i}\right) r_{i}^{n+1-k} \cdot \sum_{s=1 ; s \neq i}^{k+m} r_{s}\right.
$$

$$
\left.\cdots,(-1)^{k+m-1} \sum_{i=1}^{k+m} C_{i} D_{k}\left(r_{i}\right) r_{i}^{n+1-k} \cdot \prod_{s=1 ; s \neq i}^{k=m} r_{s}\right)
$$

The second row of $\left(e_{-n-1+i-j}^{*}\right)\left(g_{i-j}\right)$ will be the same except that the exponent of the $r_{i}$ factors in each term of the sums will be lowered by one, and similarly for the remaining rows until with the last row each $r_{i}$ appears in each sum to the power $n-k-m+2$. Since $G_{k+m}^{(i)}(z) / D_{k}(z) F_{h}(z)=G_{k+m}^{(i)}(z) \cdot \Sigma_{-\infty}^{\infty} b_{v} z^{v}=$ $\Sigma_{-\infty}^{\infty} a_{v}^{(i)} z^{v}$, it follows that

$$
\begin{aligned}
a_{v+m-1}^{(i)}= & b_{v}-b_{v+1} \sum_{s=1 ; s \neq i}^{k+m} r_{s}+b_{v+2} \sum_{1<s<t ; s, t \neq i} r_{s} r_{t} \\
& -\cdots(-1)^{k+m-1} b_{v+k+m-1} \prod_{s=1 ; s \neq i}^{k+m} r_{s} .
\end{aligned}
$$

By applying (3.6) to (3.5) it follows that the product of the matrices of (2.24) equals (3.4).

LEMMA 3.2. The determinant of the mth order matrix of (3.4) is equal to the product of two $(k+m)$ th order determinants, 

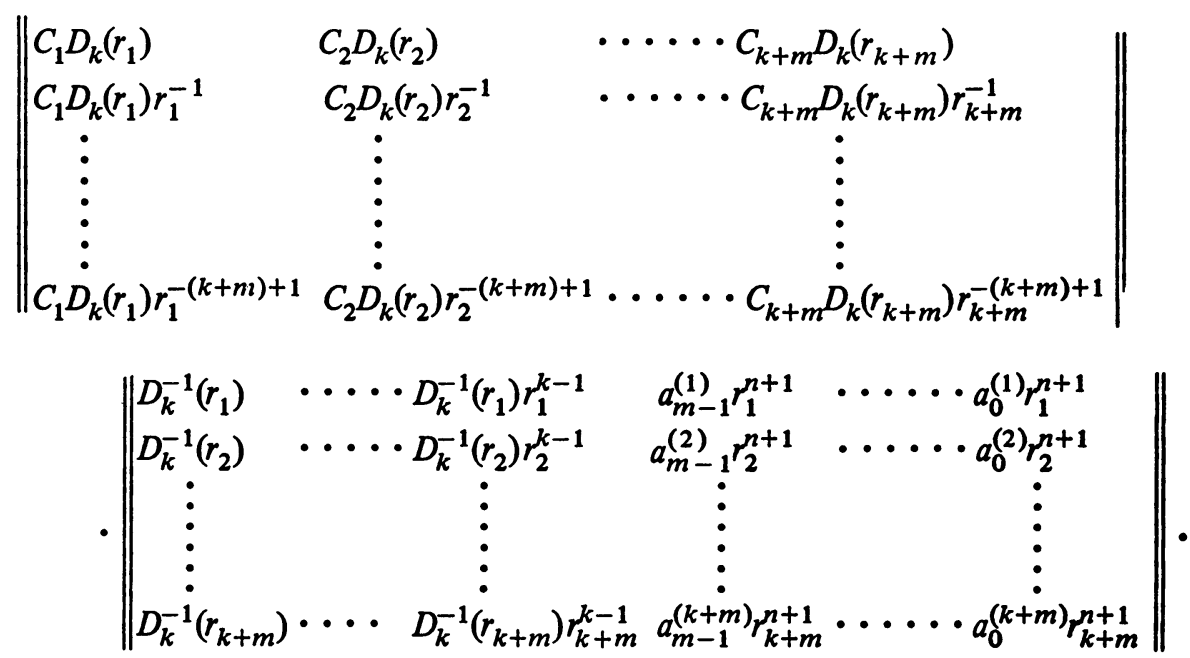

Proof. By (2.11), $\sum_{i=1}^{k+m} C_{i}=1, \sum_{i=1}^{k+m} C_{i} r_{i}^{-j}=0, j=1,2, \cdots, k+$ $m-1$. Consequently (3.7) equals

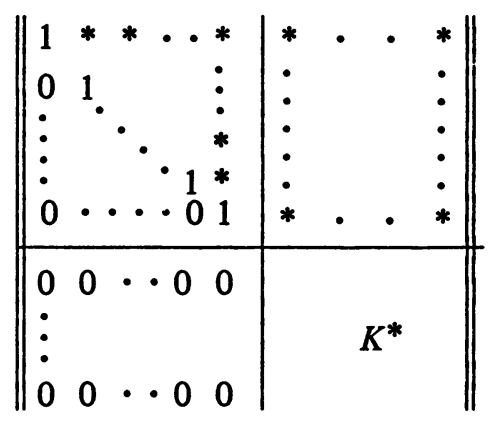

where $K^{*}$ is the matrix (3.4), and clearly (3.8) $=\left\|K^{*}\right\|$.

By Theorem $2.1, \overline{D^{n}(f)}$ equals $(-1)^{\overline{m(n+1)}}$ multiplied by the determinant of (2.24). By Lemma 3.1 and Lemma 3.2,

$$
D^{n}(f)=(-1)^{m(n+1)} \cdot[(\underline{3.7)}] .
$$

Because of the representation, except for sign, of $D^{n}(f)$ by (3.7), we are able to evaluate it. For the left-hand determinant of (3.7) is easy to evaluate, and the right-hand determinant of (3.7) may be expanded in such a way that we will be able to evaluate it.

Clearly the left-hand determinant of (3.7) equals

$$
\prod_{i=1}^{k+m} C_{i} D_{k}\left(r_{i}\right) V\left(r_{1}^{-1}, r_{2}^{-1}, \cdots, r_{k+m}^{-1}\right)
$$


where $V\left(r_{1}^{-1}, r_{2}^{-1}, \cdots, r_{k+m}^{-1}\right)$ is the Vandermonde determinant based on the numbers $r_{i}^{-1}, i=1,2, \cdots, k+m$. It can easily be shown that

$$
\begin{aligned}
\prod_{i=1}^{k+m} C_{i} & =\prod_{i=1}^{k+m} r_{i}^{k+m-1} \cdot \prod_{j \neq i}\left(r_{i}-r_{j}\right)^{-1} \\
& =(-1)^{\sigma} \prod_{i=1}^{k+m} r_{i}^{k+m-1} V^{-2}\left(r_{1}, r_{2}, \cdots, r_{k+m}\right),
\end{aligned}
$$

and that

$$
V\left(r_{1}^{-1}, r_{2}^{-1}, \cdots, r_{k+m}^{-1}\right)=(-1)^{\sigma} \prod_{i=1}^{k+m} r_{i}^{-k-m+1} V\left(r_{1}, r_{2}, \cdots, r_{k+m}\right)
$$

where $\sigma=1 / 2(k+m-1)(k+m)$. From this it follows that

$$
\underline{(3.9)}=\prod_{i=1}^{k+m} D_{k}\left(r_{i}\right) V^{-1}\left(r_{1}, r_{2}, \cdots, r_{k+m}\right) \text {. }
$$

Laplacian expansion of the right-hand determinant of (3.7) on the last $m$ columns gives us

$$
\sum_{I} \prod_{j \in \bar{I}} D_{k}^{-1}\left(r_{j}\right) V(\bar{I}) \prod_{i \in I} r_{i}^{n+1} V\left(a_{0}^{(i)}\right)
$$

The sum is taken over all subsets $I$ of $m$ integers from $(1,2, \cdots, k+m)$, and $\bar{I}=(1,2, \cdots, k+m)-I, V(\bar{I})$ is the Vandermonde determinant determined by $r_{j}, j \in \bar{I}$, and

$$
V\left(a_{0}^{(i)}\right)=\left\|\begin{array}{cccc}
a_{m-1}^{\left(i_{1}\right)} & a_{m-2}^{\left(i_{1}\right)} & \cdots & a_{0}^{\left(i_{1}\right)} \\
a_{m-1}^{\left(i_{2}\right)} & a_{m-2}^{\left(i_{2}\right)} & \cdots & a_{0}^{\left(i_{2}\right)} \\
\vdots & \vdots & \vdots \\
\vdots & \vdots & \vdots \\
a_{m-1}^{\left(i_{m}\right)} & a_{m-2}^{\left(i_{m}\right)} & \cdots & a_{0}^{\left(i_{m}\right)}
\end{array}\right\|, \quad\left(i_{1}, i_{2}, \cdots, i_{m}\right)=I
$$

LEMMA 3.3. $V\left(a_{0}^{(i)}\right)=V(I) \cdot\left\|\left(a_{s-t}^{I}\right)_{s, t=1}^{m}\right\|$.

Proof. As before, $V(I)$ is the Vandermonde determinant determined by $I$, and $\left(a_{s-t}^{I}\right)_{s, t=1}^{m}$ is the $m$ th order Toeplitz matrix generated by the function

$$
G_{k+m}^{I}(z) / D_{k}(z) \cdot F_{h}(z)=\sum_{-\infty}^{\infty} a_{v}^{I} z^{v}
$$

defined by (3.1) and (3.3). We give a demonstration of this for

$$
\left\|\begin{array}{cccc}
a_{m-1}^{(1)} & a_{m-2}^{(1)} & \cdots & a_{0}^{(1)} \\
a_{m-1}^{(2)} & a_{m-2}^{(2)} & \cdots & a_{0}^{(2)} \\
\vdots & \vdots & \vdots \\
a_{m-1}^{(m)} & a_{m-2}^{(m)} & \cdots & a_{0}^{(m)}
\end{array}\right\|
$$


By the convention (3.3), if $S$ is any subset of the set $(1,2, \cdots, k+m)$ with $s$ elements, then

$$
a_{v+m-S}^{S}=b_{v}-b_{v+1} \sum_{i \notin S} r_{i}+\cdots+(-1)^{k+m-s} b_{v+k+m-s} \prod_{i \notin S} r_{i} .
$$

So we can easily verify that $a_{v+m-1}^{(i)}=a_{v+m-2}^{(i, j)}-r_{j} a_{v+m-1}^{(i, j)}$ and that

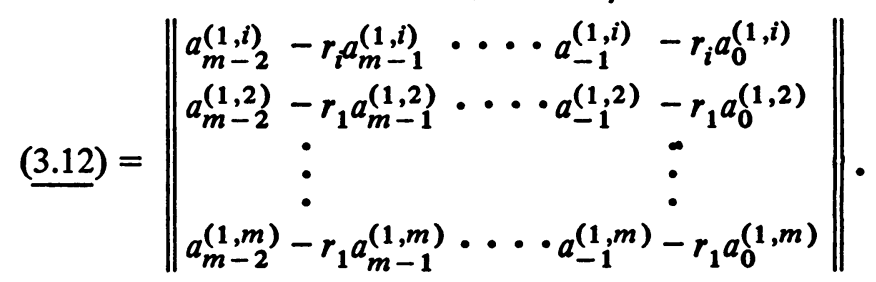

Letting $i$ alternately be equal to $2,3, \cdots, m$, and subtracting the first row from each of the others, and rewriting the first row in its original form, we get that the right-hand side of (3.13) equals

$$
\left\|\begin{array}{ccc}
a_{m-1}^{(1)} & \cdots & a_{0}^{(1)} \\
a_{m-1}^{(1,2)} & \cdots & a_{0}^{(1,2)} \\
\vdots & & \vdots \\
a_{m-1}^{(1, m)} & \cdots & a_{0}^{(1, m)}
\end{array}\right\|\left(r_{2}-r_{1}\right)\left(r_{3}-r_{1}\right) \cdots\left(r_{m}-r_{1}\right)
$$

Since $a_{v}^{(1)}=a_{v-1}^{(1,2)}-r_{2} a_{v}^{(1,2)}$, if we add $r_{2}$ times the second row to the first row in (3.14), we obtain the result that (3.12) equals

$$
\left\|\begin{array}{ccc}
a_{m-2}^{(1,2)} & \cdots & a_{-1}^{(1,2)} \\
a_{m-1}^{(1,2)} & \cdots & a_{0}^{(1,2)} \\
\vdots & \vdots \\
a_{m-1}^{(1, m)} & \cdots & \vdots \\
a_{0}^{(1, m)}
\end{array}\right\|\left(r_{2}-r_{1}\right) \cdots\left(r_{m}-r_{1}\right)
$$

Similarly using the second row as a pivot row to reduce the rows below it, we can verify that the determinant in (3.15) equals

$$
\left\|\begin{array}{ccc}
a_{m-2}^{(1,2)} & \cdots & a_{-1}^{(1,2)} \\
a_{m-1}^{(1,2)} & \cdots & a_{0}^{(1,2)} \\
a_{m-1}^{(1,2,3)} & \cdots & a_{0}^{(1,2,3)} \\
\vdots & \vdots \\
\vdots & \vdots \\
a_{m-1}^{(1,2, m)} & \cdots \cdots & a_{0}^{(1,2, m)}
\end{array}\right\|\left(r_{3}-r_{2}\right)\left(r_{4}-r_{2}\right) \cdots\left(r_{m}-r_{2}\right)
$$


Adding $r_{3}$ times the third row to the second row, and subsequently $r_{3}$ times the second row to the first row, we reduce (3.16) to

$$
\left\|\begin{array}{ccc}
a_{m-3}^{(1,2,3)} & \cdots & a_{-2}^{(1,2,3)} \\
a_{m-2}^{(1,2,3)} & \cdots & a_{-1}^{(1,2,3)} \\
a_{m-1}^{(1,2,3)} & \cdots & a_{0}^{(1,2,3)} \\
\vdots & \vdots \\
\vdots & \vdots \\
a_{m-1}^{(1,2, m)} & \cdots & a_{0}^{(1,2, m)}
\end{array}\right\|\left(r_{3}-r_{2}\right) \cdots\left(r_{m}-r_{2}\right)
$$

Continuing in this manner, successively using the third, fourth, etc. rows as pivot rows and factoring out the appropriate factors, we prove that for $I=(1,2, \cdots, m)$,

$$
(\underline{3.12})=V(I)\left\|\left(a_{s-t}^{I}\right)_{s, t=1}^{m}\right\| .
$$

The general proof follows as above.

Consequently, by (3.8), (3.10), (3.11) and Lemma 3.3,

$$
\begin{aligned}
D^{n}(f)= & (-1)^{m(n+1)} \prod_{i=1}^{k+m} D_{k}\left(r_{i}\right) V^{-1}\left(r_{1}, r_{2}, \cdots, r_{k+m}\right) \\
& \cdot\left[\sum_{I} \prod_{j \in \bar{I}} D_{k}^{-1}\left(r_{j}\right) V(\bar{I}) \cdot \prod_{i \in I} r_{i}^{n+1} V(I)\left\|\left(a_{s-t}^{I}\right)_{s, t=1}^{m}\right\|\right] \\
= & (-1)^{m(n+1)} \sum_{I} \prod_{i \in I ; j \in \bar{I}} r_{i}^{n+1} D_{k}\left(r_{i}\right) \cdot\left\|\left(a_{s-t}^{I}\right)_{s, t=1}^{m}\right\|\left(r_{i}-r_{j}\right)^{-1},
\end{aligned}
$$

where $I$ runs over all subsets of order $m$ of the set $(1,2, \cdots, k+m)$, and $\bar{I}=$ $(1,2, \cdots, k+m)-I$.

LEMMA 3.4. $\left\|\left(a_{s-t}^{I}\right)_{s, t=1}^{m}\right\|=\left\|\left(a_{s-t}^{I}\right)_{s, t=1}^{h}\right\|$.

Proof. By (3.3), $\Sigma_{-\infty}^{\infty} a_{v} z^{v}=G_{k+m}^{I}(z) / D_{k}(z) F_{h}(z)$, and $G_{k+m}^{I}(z)=$ $\Pi_{i=1 ; i \notin I}^{k+m}\left(z-r_{i}\right)$, a monic $k$ th degree polynomial. By $(2.3), D_{k}(z)$ is also a monic $k$ th degree polynomial. Therefore the power series expansion of

$$
G_{k+m}^{I}(z) / D_{k}(z)=\left(\sum_{-\infty}^{\infty} a_{v}^{I} z^{v}\right) \cdot F_{h}(z) \text { around } z=\infty
$$

commences with the constant term 1. By (2.5), $F_{h}(z)=\sum_{i=0}^{h} f_{i}^{*} z^{i}$ with $f_{0}^{*}=1$. By (3.18) it follows that 


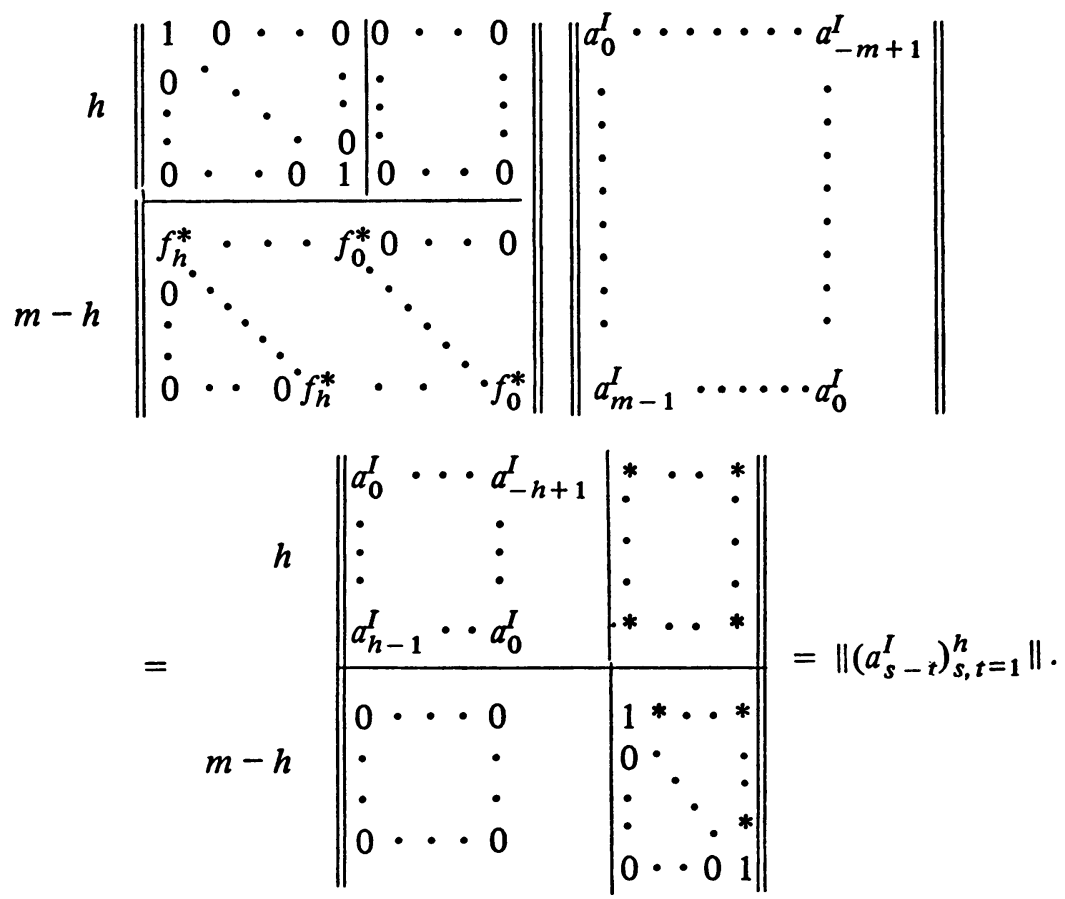

By applying the results of this lemma to (3.17), we have derived the following identity for $D^{n}(f)$.

$$
D^{n}(f)=(-1)^{m(n+1)} \sum_{I} \prod_{i \in I ; j \in \bar{I}} r_{i}^{n+1} D_{k}\left(r_{i}\right) \cdot\left\|\left(a_{s-t}^{I}\right)_{s, t=1}^{h}\right\|\left(r_{i}-r_{j}\right)^{-1}
$$

where $I$ runs over all subsets of order $m$ of the set $(1,2, \cdots, k+m)$, and $\bar{I}=$ $(1,2, \cdots, k+m)-I$. The only factors that are not explicitly evaluated are the $h$ th order determinants $\left\|\left(a_{s-t}^{I}\right)_{s, t=1}^{h}\right\|$. Since the $a_{s}^{I}$ 's are generated by the function

and by (3.1)

$$
G_{k+m}^{I}(z) / D_{k}(z) F_{h}(z)=\sum_{-\infty}^{\infty} a_{v}^{I} z^{v}
$$

$$
G_{k+m}^{I}(z)=\prod_{j \in \bar{I}}\left(z-r_{j}\right)
$$

each $a_{s}^{I}$ is a polynomial in the $r_{j}$ 's, $j \in \bar{I}$. Consequently each determinant $\left\|\left(a_{s-t}^{I}\right)_{s, t=1}^{h}\right\|$ is a polynomial at most of $h$ th degree in each of the $r_{j}$ 's, $j \in \bar{I}$.

Lemma 3.5. $\left\|\left(a_{s-t}\right)_{s, t=1}^{h}\right\|=C \Pi_{j \in \bar{I}} F_{h}\left(r_{j}\right)$ where $C$ is a constant that is independent of the $r_{j}$ 's, $j \in \bar{I}$.

Proof. The factor $\Pi_{j \in \bar{I}} F_{h}\left(r_{j}\right)$ is of exact degree $h$ in each of the $r_{j}$ 's. So by the remarks immediately preceding the lemma we can conclude that $C$ is a constant which is independent of the $r_{j}$ 's. Direct evaluation of the determinants $\left\|\left(a_{s-t}^{I}\right)_{s, t=1}^{h}\right\|$ seems to be difficult. Consequently we approach the problem in- 
directly. We made the remark in the proof of Lemma 2.3 that the reduction of $D^{n}(f)$ to the $m$ th order determinant (2.24) which led to the identity (3.19) was effected by multiplication of $D^{n}(f)$ by determinants (2.17) and (2.18) which are asymmetric. We indicated that another reduction is possible.

Define the Laurent series $\Sigma_{-\infty}^{\infty} \hat{a}_{v} z^{v}$ by

so that

$$
(-1)^{k+m} \prod_{i=1}^{k+m} r_{i}^{-1} \cdot \sum_{-\infty}^{\infty} a_{v} z^{v}=\sum_{-\infty}^{\infty} \hat{a}_{v} z^{v}
$$

$$
(-1)^{k+m} \prod_{i=1}^{k+m} r_{i}^{-1} \cdot a_{v}=\hat{a}_{v}
$$

Since each $r_{i}$ is assumed to be nonzero, multiplication by $\Pi_{i=1}^{k+m} r_{i}^{-1}$ is possible. Clearly if $\hat{f}(z)=(-1)^{k+m} \Pi_{i=1}^{k+m} r_{i}^{-1} \cdot f(z)$, then $\hat{f}(z)=\Sigma_{-\infty}^{\infty} \hat{a}_{v} z^{v}$ in the annulus $A$. Let $D^{n}(\hat{f})$ be the $(n+1)$ st order determinant generated by the $\hat{a}_{v}$ 's. We may reduce $D^{n}(\hat{f})$ to a determinant of fixed order exactly as we did in $\S 2$ for $D^{n}(f)$. $\hat{f}(z)$ is

Since the numerator of $f(z)$ is $G_{k+m}(z)=\prod_{i=1}^{k+m}\left(z-r_{i}\right)$, the numerator of

$$
\begin{aligned}
(-1)^{k+m} r_{i}^{-1} G_{k+m}(z) & =\prod_{i=1}^{k+m}\left(1-r_{i}^{-1} z\right), \text { and } \\
\hat{f}(z) & =\prod_{i=1}^{k+m}\left(1-r_{i}^{-1} z\right) / D_{k}(z) F_{h}(z) .
\end{aligned}
$$

Let us multiply $D^{n}(\hat{f})$ on the left by the upper triangular determinant $D^{n}\left(z^{-k} D_{k}(z)\right)=1$, and on the right by the lower triangular determinant $D^{n}\left(F_{h}(z) / \Pi_{i=1}^{k+m}\left(1-r_{i}^{-1} z\right)\right)=1$. Reduction of the determinant $D^{n}(\hat{f})$ by the techniques of $\S 2$ results in reducing $D^{n}(\hat{f})$ to a determinant of order $k$ similar to (2.24). Consequent evaluation of this $k$ th order determinant exactly as we have evaluated $D^{n}(f)$ results in the identity

$$
\begin{aligned}
D^{n}(\hat{f})=(-1)^{k(n+1)} \sum_{\bar{I}} \prod_{j \in \bar{I} ; i \in I} r_{j}^{-n-1-m} F_{h}\left(r_{j}\right) \\
\qquad\left\|\left(\hat{a}_{-k 4 s-t}^{\bar{I}}\right)_{s, t=1}^{k}\right\|\left(r_{j}^{-1}-r_{i}^{-1}\right)^{-1},
\end{aligned}
$$

where $\bar{I}$ runs over all subsets of order $k$ of the set $(1,2, \cdots, k+m)$, and $I=$ $(1, \cdots, k+m)-\bar{I}$, and the coefficients $\hat{a}_{s}^{\bar{I}}$ are generated by the function

$$
\prod_{i \in I}\left(1-r_{i}^{-1} z\right) / D_{k}(z) F_{h}(z)=\sum_{-\infty}^{\infty} \hat{a}_{v}^{\bar{T}} z^{v}
$$

A comparison of the identity for $D^{n}(\hat{f})$ (3.22) with the identity for $D^{n}(f)$ (3.19) results in some apparent discrepancies. In (3.19) appear the factors $\Pi_{i \in r_{i}} r^{n+1} D_{k}\left(r_{i}\right)$, of degree $n+k+1$ in each of the $r_{i}$ 's. In (3.22) appear the factors $\Pi_{j \in \bar{I}} r_{j}^{-n-1-m} F_{h}\left(r_{j}\right)$. Since $F_{h}(0) \neq 0$, and $m \geqslant h$, these factors are of degree $n+m+1$ in each of the $r_{j}^{-1}$, s which is what is desired. We note that the coef- 
ficient $\hat{a}_{-k}^{I}$ lies on the main diagonal of the unevaluated determinantal factors appearing in (3.22) rather than $\hat{a}_{0}^{\bar{I}}$. This occurs because of differences which arise in simplifying the determinants arising in the alternative approach vis-à-vis the simplification of the determinants appearing in Lemma 3.2.

By (3.20),

$$
(-1)^{(k+m)(n+1)} \prod_{i=1}^{k+m} r_{i}^{n+1} D^{n}(\hat{f})=D^{n}(f)
$$

Because

and

$$
(-1)^{(k+m)(n+1)} \cdot(-1)^{k(n+1)}=(-1)^{m(n+1)}
$$

$$
\prod_{j \in \bar{I} ; i \in I}\left(r_{j}^{-1}-r_{i}^{-1}\right)^{-1}=\prod_{i \in I ; j \in \bar{I}} r_{i}^{k} r_{j}^{m}\left(r_{i}-r_{j}\right)^{-1},
$$

we have, after carrying out the multiplication indicated on the left-hand side of (3.23), that

$$
\begin{aligned}
& (-1)^{m(n+1)} \sum_{I} \prod_{i \in I ; j \in \bar{I}} r_{i}^{n+1} \cdot r_{i}^{k}\left\|\left(\hat{a}_{-k+s-t}^{\bar{I}}\right)_{s, t=1}^{k}\right\| \cdot F_{h}\left(r_{j}\right)\left(r_{i}-r_{j}\right)^{-1} \\
& =(-1)^{m(n+1)} \sum_{I} \prod_{i \in I ; j \in \bar{I}} r_{i}^{n+1} \cdot D_{k}\left(r_{i}\right)\left\|\left(a_{s-t}^{I}\right)_{s, t=1}^{h}\right\|\left(r_{i}-r_{j}\right)^{-1} .
\end{aligned}
$$

Because the $r_{i}$ may be chosen independently of one another and the above equality holds for all $n$, we may equate corresponding terms which are defined by the same set of indices $I$. From this it follows that, for each $I$,

Consequently

$$
\begin{aligned}
\prod_{i \in I ; j \in \bar{I}} r_{i}^{n+1} & \cdot\left[r_{i}^{k}\left\|\left(\hat{a}_{-k+s-t}^{\bar{I}}\right)_{s, t=1}^{k}\right\|\right] \cdot F_{h}\left(r_{j}\right)\left(r_{i}-r_{j}\right)^{-1} \\
& =\prod_{i \in I ; j \in \bar{I}} r_{i}^{n+1} D_{k}\left(r_{i}\right)\left\|\left(a_{-s-t}^{I}\right)_{s, t=1}^{h}\right\|\left(r_{i}-r_{j}\right)^{-1}
\end{aligned}
$$

$$
\prod_{i \in I ; j \in \bar{I}}\left[r_{i}^{k}\left\|\left(\hat{a}_{-k+s-t}^{I}\right)_{s, t=1}^{k}\right\|\right] \cdot F_{h}\left(r_{j}\right)=\prod_{i \in I ; j \in \bar{I}} D_{k}\left(r_{i}\right) \cdot\left\|\left(a_{s-t}^{I}\right)_{s, t=1}^{h}\right\| .
$$

But, as we remarked immediately preceding this lemma, $\left\|\left(a_{s-t}^{I}\right)_{1}^{h}\right\|$ is a polynomial at most of degree $h$ in each of the $r_{j}$ 's, $j \in \bar{I}$. Similarly, since the $\hat{a}_{v}^{\bar{I}}$, s are functions of the $r_{i}^{-1}$ 's, $i \in I, \Pi_{i \in I} r_{i}^{k}\left\|\left(\hat{a}_{-k+s-t}^{\bar{I}}\right)_{1}^{k}\right\|$ is a polynomial at most of degree $k$ in each $r_{i}, i \in I$. We may conclude from these observations and (3.24) that

$$
\left\|\left(a_{s-t}^{I}\right)_{s, t=1}^{h}\right\|=C \prod_{j \in \bar{I}} F_{h}\left(r_{j}\right)
$$

and

$$
\coprod_{i \in I} r_{i}^{k}\left\|\left(\hat{a}_{-k+s-t}^{\bar{I}}\right)_{s, t=1}^{h}\right\|=C \prod_{i \in I} D_{k}\left(r_{i}\right) .
$$

This concludes the proof of Lemma 3.5.

We have almost completed the evaluation of $D^{n}(f)$. Applying the results of Lemma 3.5 to (3.19), the identity for $D^{n}(f)$ assumes the form 
(3.26) $D^{n}(f)=(-1)^{m(n+1)} \cdot C \cdot \sum_{I} \prod_{i \in I ; j \in \bar{I}} r_{i}^{n+1} D_{k}\left(r_{i}\right) F_{h}\left(r_{j}\right)\left(r_{i}-r_{j}\right)^{-1}$,

where $I$ runs over all subsets of order $m$ of the set $(1,2, \cdots, k+m)$ and $\bar{I}=$ $(1,2, \cdots, k+m)-I$. Only the constant term $C$ remains to be determined.

Lemma 3.6. $C=\Pi_{s \in K ; t \in H} \rho_{t}^{k} /\left(\rho_{t}-\delta_{s}\right)$ where $K=(1, \cdots, k)$ and $H=(1, \cdots, h)$.

Proof. Because the constant $C$ is independent of the roots $r_{i}$ of the polynomial $G_{k+m}(z)$, we may choose the roots and thereby the polynomial $G_{k+m}(z)$ in such a way that the constant $C$ will be determined. In particular we may assume that $m=h$. Choose the roots $r_{i}, i=1, \cdots, k$, to lie respectively within an $\epsilon$-neighborhood of the roots $\delta_{s}, s=1, \cdots, k$, of $D_{k}(z)$ and the roots $r_{i}, i=k+$ $1, \cdots, k+h$, to lie within an $\epsilon$-neighborhood of the roots $\rho_{t}, t=1, \cdots, h$, of $F_{h}(z)$ but such that $\left|r_{i}-r_{j}\right| \geqslant O(\epsilon)$ where $\epsilon$ is a small positive number. Let $I_{0}=(k+1, \cdots, k+h), \bar{I}_{0}=(1, \cdots, k)$. We wish to show for all $I \neq I_{0}$ that if the roots $r_{i}$ tend to the roots $\delta_{s}$ and $\rho_{t}$ of $D_{k}(z)$ and $F_{h}(z)$ respectively, then

$$
\prod_{i \in I ; j \in \bar{I}} D_{k}\left(r_{i}\right) F_{h}\left(r_{j}\right) /\left(r_{i}-r_{j}\right)
$$

tends to zero. This would be obvious except for the possibility that $D_{k}(z)$ and $F_{h}(z)$ may have multiple roots and so for some $i, j, r_{i}-r_{j} \rightarrow 0$.

For $I \neq I_{0}, I$ and $\bar{I}$ may each be written as the union of a nonempty disjoint pair of sets as follows. Let $I=I_{0}^{*} \cup \bar{I}_{0}^{*}$ and $\bar{I}=I_{0}^{* *} \cup \bar{I}_{0}^{* *}$ where $I_{0}^{*}$, $I_{0}^{* *} \subset I_{0}$, and $\bar{I}_{0}, \bar{I}_{0}^{* *} \subset \bar{I}_{0}$. But then

$$
\begin{aligned}
&(\underline{3.27})=\prod_{i \in I_{0}^{*} ; j \in I_{0}^{* *}} \frac{D_{k}\left(r_{i}\right) F_{h}\left(r_{j}\right)}{\left(r_{i}-r_{j}\right)} \cdot \prod_{i \in \bar{I}_{0}^{*}, i \in \bar{I}_{0}^{* *}} \frac{D_{k}\left(r_{i}\right) F_{h}\left(r_{j}\right)}{\left(r_{i}-r_{j}\right)} \\
& \cdot \prod_{i \in I_{0}^{*} ; j \in \bar{I}_{0}^{* *}} \frac{D_{k}\left(r_{i}\right) F_{h}\left(r_{j}\right)}{\left(r_{i}-r_{j}\right)} \cdot \prod_{i \in I_{0}^{*} ; j \in \bar{I}_{0}^{* *}} \frac{D_{k}\left(r_{i}\right) F_{h}\left(r_{j}\right)}{\left(r_{i}-r_{j}\right)} \\
&=\hat{B} \cdot \hat{C} \cdot \hat{D} \cdot \hat{E}, \text { say. }
\end{aligned}
$$

From the manner in which the roots $r_{i}$ are chosen,

$$
\prod_{i \in I_{0}^{*} ; j \in I_{0}^{* *}} \frac{F_{h}\left(r_{j}\right)}{\left(r_{i}-r_{j}\right)}, \quad \prod_{i \in \bar{I}_{0}^{*} ; j \in \bar{I}_{0}^{* * *}} \frac{D_{k}\left(r_{i}\right)}{\left(r_{i}-r_{j}\right)}, \prod_{i \in I_{0}^{*} ; j \in \bar{I}_{0}^{* *}} \frac{1}{\left(r_{i}-r_{j}\right)}
$$

$=O(1)$, since the roots $\delta_{S}$ and $\rho_{t}$ are separated by the annulus $A$. So $\hat{B}=\hat{C}=$ $\hat{D}=O(1)$. But necessarily $\hat{D}=O(\epsilon)$. Consequently (3.27) $\rightarrow 0$ as the roots $r_{i}$ tend to the appropriate limits. With the same limits,

$$
\lim \prod_{i \in I_{0} ; j \in \bar{I}_{0}} \frac{D_{k}\left(r_{i}\right) F_{h}\left(r_{j}\right)}{\left(r_{i}-r_{j}\right)}=\prod_{s \in K ; t \in H} \frac{D_{k}\left(\rho_{t}\right) F_{h}\left(\delta_{s}\right)}{\left(\rho_{t}-\delta_{s}\right)} .
$$


So the limit of the right-hand side of (3.26) equals

$$
(-1)^{h(n+1)} \cdot C \cdot \prod_{s \in K ; t \in H} \rho_{t}^{n+1} D_{k}\left(\rho_{t}\right) F_{h}\left(\delta_{s}\right) /\left(\rho_{t}-\delta_{s}\right) .
$$

Moreover it is clear that

$$
\begin{aligned}
f(z) & =\prod_{i=1}^{k+h}\left(z-r_{i}\right) /\left[\prod_{s=1}^{k}\left(z-\delta_{s}\right) \prod_{t=1}^{h}\left(1-\rho_{t}^{-1} z\right)\right] \\
& =\frac{\left[(-1)^{h} \Pi_{i=1}^{k}\left(z-r_{i}\right) \Pi_{i=k+1}^{k+h} r_{i}\left(1-r_{i}^{-1} z\right)\right]}{\left[\Pi_{s=1}^{k}\left(z-\delta_{s}\right) \Pi_{t=1}^{h}\left(1-\rho_{t}^{-1} z\right)\right]}
\end{aligned}
$$

tends to $(-1)^{h} \Pi_{t \in H} \rho_{t}$, a constant.

For each $n, D^{n}(f)$ varies continuously with the coefficients $a_{m}$ of the Laurent series representation of $f$ and these coefficients vary continuously with the roots of $G_{k+h}(z)$. Consequently

From (3.26) and (3.29)

$$
\lim D^{n}(f)=(-1)^{h(n+1)} \prod_{t \in H} \rho_{t}^{n+1}
$$

$$
\begin{aligned}
(-1)^{h(n+1)} \prod_{t \in H} \rho_{t}^{n+1}= & (-1)^{h(n+1)} \cdot C \\
& \cdot \prod_{s \in K ; t \in H} \rho_{t}^{n+1} D_{k}\left(\rho_{t}\right) F_{h}\left(\delta_{s}\right) /\left(\rho_{t}-\delta_{s}\right),
\end{aligned}
$$

and so

$$
C=\prod_{s \in K ; t \in H}\left(\rho_{t}-\delta_{s}\right) / D_{k}\left(\rho_{t}\right) F_{h}\left(\delta_{s}\right) .
$$

But $D_{k}\left(\rho_{t}\right)=\Pi_{s \in K}\left(\rho_{t}-\delta_{s}\right)$ and $F_{h}\left(\delta_{s}\right)=\Pi_{t \in H}\left(1-\rho_{t}^{-1} \delta_{s}\right)=\Pi_{t \in H} \rho_{t}^{-1}\left(\rho_{t}-\delta_{s}\right)$. Consequently

$$
C=\prod_{s \in K ; t \in H} \frac{\rho_{t}^{k}\left(\rho_{t}-\delta_{s}\right)}{\left(\rho_{t}-\delta_{s}\right)^{2}}=\prod_{s \in K ; t \in H} \frac{\rho_{t}^{k}}{\left(\rho_{t}-\delta_{s}\right)} .
$$

We are now in the position to prove the following theorem.

THEOREM 3.1. Let $f(z)=G_{k+m}(z) / D_{k}(z) F_{h}(z)$ where $G_{k+m}(z), D_{k}(z)$, and $F_{h}(z)$ satisfy the conditions of Proposition 1.1. Assume, in addition, that the zeros of $G_{k+m}(z)$ are distinct and not equal to zero, and that the coefficient of the $z^{k+m}$ term of $G_{k+m}(z)$ is equal to 1 . Then

$$
D^{n}(f)=(-1)^{m(n+1)} \sum_{\substack { I \\
\begin{subarray}{c}{i \in I, s \in K \\
j \in I, t \in H{ I \\
\begin{subarray} { c } { i \in I , s \in K \\
j \in I , t \in H } }\end{subarray}} r_{i}^{n+1}\left[\frac{\left(r_{i}-\delta_{s}\right)\left(\rho_{t}-r_{j}\right)}{\left(r_{i}-r_{j}\right)\left(\rho_{t}-\delta_{s}\right)}\right]
$$

where I runs over all subsets of order $m$ of the set $(1, \cdots, k+m), \bar{I}=(1, \cdots$, $k+m)-I, K=(1, \cdots, k)$, and $H=(1, \cdots, h)$.

PROoF. By definition of $D_{k}(z)$ and $F_{h}(z), D_{k}\left(r_{i}\right)=\Pi_{s \in K}\left(r_{i}-\delta_{s}\right)$, and 
$F_{h}\left(r_{j}\right)=\Pi_{t \in H} \rho_{t}^{-1}\left(\rho_{t}-r_{j}\right)$. So by Lemma 3.6 and (3.26),

$$
\begin{aligned}
D^{n}(f) & =(-1)^{m(n+1)} \sum_{I} \prod_{\substack{i \in I, s \in K \\
j \in \bar{I}_{,}, t \in H}} \frac{r_{i}^{n+1} \rho_{t}^{k}\left(r_{i}-\delta_{s}\right) \rho_{t}^{-k}\left(\rho_{t}-r_{j}\right)}{\left(r_{i}-r_{j}\right)\left(\rho_{t}-\delta_{s}\right)} \\
& =(-1)^{m(n+1)} \sum_{\substack{I \\
I}} \prod_{\substack{i \in I_{i} \\
j \in I_{,}, t \in H}} \frac{r_{i}^{n+1}\left(r_{i}-\delta_{s}\right)\left(\rho_{t}-r_{j}\right)}{\left(r_{i}-r_{j}\right)\left(\rho_{t}-\delta_{s}\right)},
\end{aligned}
$$

which is the conclusion of the theorem.

4. Applications of the identity for $D^{n}(f)$. Let $A$ be the annulus defined by (1.1). Let $G_{s}(z), D_{k}(z), F_{h}(z)$ be polynomials having no common factors of exact degree $s, k$, and $h$ respectively. We assume $D_{k}(z)$ and $F_{h}(z)$ satisfy the conditions required by Proposition 1.1 and are expressed as in (2.3) and (2.5) respectively. We impose no conditions upon $G_{s}(z)$. We assume that $k \geqslant 1$, and if $h=0$ that $s \geqslant k+1$.

It follows that

$$
f(z)-\lambda=\frac{G_{s}(z)-\lambda D_{k}(z) F_{h}(z)}{D_{k}(z) F_{h}(z)}=\frac{G_{k+m}(\lambda, z)}{D_{k}(z) F_{h}(z)}
$$

where $G_{k+m}(\lambda, z)=G_{s}(z)-\lambda D_{k}(z) F_{h}(z)$ and $k+m=\max (s, k+h)$.

If $s \leqslant k+h$ there is one value of $\lambda$ for which $G_{k+m}(\lambda, z)$ has less than $k+m$ roots, otherwise $G_{k+m}(\lambda, z)$ is of exact degree $k+m$ and satisfies the hypothesis of Proposition 1.1. Thus the Laurent series expansion (1.3) of $f(z)$ $-\lambda$ in $A$ generates matrices $T_{n}(f-\lambda)$ which are not triangular.

From the theory of algebraic functions [2, pp. 103-104] the set of values of $\lambda$ for which $G_{k+m}(\lambda, z)=0$ has multiple roots, has $z=0$ as a root, or has less than $k+m$ roots is a finite set. For all other $\lambda$ we denote the roots of $G_{k+m}(\lambda, z)=0$ by $r_{i}(\lambda), i=1, \cdots, k+m$, and

$$
f(z)-\lambda=c(\lambda) \prod_{i=1}^{k+m}\left(z-r_{i}(\lambda)\right) / D_{k}(z) F_{h}(z),
$$

$c(\lambda)=a, a-\lambda b$, or $-b$ according to whether $s>k+h, s=k+h$, or $s<k+h$. Note, $a$ is the coefficient of $z^{s}$ of $G_{s}(z)$, and $b$ is the coefficient of $z^{k+h}$ of $D_{k}(z) \cdot F_{h}(z)$.

By Theorem 3.1 and the first paragraph of $\S 2$

(4.1) $D^{n}(f-\lambda)=\left[(-1)^{m} c(\lambda)\right]^{n+1} \sum_{\substack { I \\ \begin{subarray}{c}{i \in I_{1}, s \in K \\ j \in \overline{,}, t \in H{ I \\ \begin{subarray} { c } { i \in I _ { 1 } , s \in K \\ j \in \overline { , } , t \in H } }\end{subarray}} r_{i}(\lambda)^{n+1} \frac{\left(r_{i}(\lambda)-\delta_{s}\right)\left(\rho_{t}-r_{j}(\lambda)\right)}{\left(r_{i}(\lambda)-r_{j}(\lambda)\right)\left(\rho_{t}-\delta_{s}\right)}$.

Assume that for fixed $\lambda$ the roots $r_{i}(\lambda)$ are indexed by increasing modulus, so that $\left|r_{1}(\lambda)\right| \leqslant\left|r_{2}(\lambda)\right| \leqslant \cdots \leqslant \mid r_{k+m}(\lambda) l$. We define the set $C$ to be the set 


$$
C=\left\{\lambda:\left|r_{k}(\lambda)\right|=\left|r_{k+1}(\lambda)\right|\right\} .
$$

The analysis provided in [3] and [4] shows that the set $C$ is bounded, contains no isolated points, and consists of a finite union of closed analytic arcs. In addition the arguments of J. L. Ullman [5] may be employed to show that the set $C$ is connected. Since the set $C$ contains no isolated points and the set of $\lambda$ 's for which the identity (4.1) does not hold is a finite set, the techniques of Schmidt and Spitzer allow us to make the identification of the limit set $B$ of the eigenvalues $\sigma_{n}$ with the set $C$ defined above.

A related question is the following. Define a sequence of measures $\alpha_{n}$,

$$
\alpha_{n}(E)=(n+1)^{-1} \sum_{\lambda_{n i} \in E} 1,
$$

where $\lambda_{n i} \in \sigma_{n}$, and $E$ is an arbitrary set in the $\lambda$-plane. Let $\alpha$ be any weak limit of the measures $\alpha_{n}$. It will be shown in a later paper that the limit measure $\alpha$ is unique and has at most two atoms. The rational functions $f$ for which $\alpha$ has atoms will be characterized and the weight of the atoms determined.

\section{REFERENCES}

1. G. Baxter and P. Schmidt, Determinants of a certain class of non-Hermitian Toeplitz matrices, Math. Scand. 9 (1961), 122-128.

2. E. Hille, Analytic function theory. Vol. 2, Introduction to Higher Math., Ginn, Boston, Mass., 1962. MR 34 \#1490.

3. I. I. Hirschman, Jr., The spectra of certain Toeplitz matrices, Illinois J. Math. 11 (1967), 145-159. MR 34 \#4905.

4. P. Schmidt and F. Spitzer, The Toeplitz matrices of an arbitrary Laurent polynomial, Math. Scand. 8 (1960), 15-38. MR 23 \#A1977.

5. J. L. Ullman, A problem of Schmidt and Spitzer, Bull. Amer. Math. Soc. 73 (1967), 883-885. MR 36 \#3056.

DEPARTMENT OF MATHEMATICS, UNIVERSITY OF MICHIGAN, ANN ARBOR, MICHIGAN 48104 